\section{Efficacy and safety of Camosunate for the treatment of uncomplicated malaria in the University of Benin Teaching Hospital, Benin City, Nigeria}

\author{
Damien Uyagu, ${ }^{1}$ Augustine Omoigberale, ${ }^{2}$ \\ Paul Dienye ${ }^{3}$ \\ 'Department of Family Medicine, \\ University of Benin Teaching Hospital, \\ Benin City; ' 2Department of Child Health, \\ University of Benin Teaching Hospital, \\ Benin City; ${ }^{3}$ Department of Family \\ Medicine, University of Port Harcourt \\ Teaching Hospital, Port Harcourt, Nigeria
}

\section{Abstract}

In Nigeria, nearly 110 million clinical cases of malaria are diagnosed per year, thus being a major public health problem. The problems of resistance resulted in the introduction of the artemisinin based combinations (ACT) by the WHO. Artesunate and amodiaquine (AS+AQ) is at present the world's second most widely used ACT. This study is an assessment of the efficacy and safety of Camosunate (a brand of AS+AQ; Geneith Pharmaceutical Ltd., Oshodi, Lagos) in the treatment of uncomplicated malaria conducted at the University of Benin Teaching Hospital (UBTH). A cross-sectional assessment of the efficacy and safety of Camosunate was conducted over a period of one year using 120 patients selected after stratification, by random sampling technique. All recruited patients had slide-proven uncomplicated malaria and were followed up for 28 days on commencement of Camosunate. Data was collected using a structured intervieweradministered questionnaire and was analysed using SPSS version 15. The overall efficacy of Camosunate was found to be $95.8 \%$. Treatment was well tolerated as testified by the fact that there was no case withdrawal due to adverse drug reaction (ADR) or treatment emergent signs and symptoms (TESS). Also no evidence of toxicity was recorded. Camosunate is highly efficacious and well tolerated in this area of Nigeria and justifies its use as a first line treatment for uncomplicated malaria.

\section{Introduction}

In Nigeria, nearly 110 million clinical cases of malaria are diagnosed per year, translating to about $50 \%$ of the adult population experienc- ing at least one malaria episode per year, while young children can have up to 2-4 attacks of malaria annually, ${ }_{1}^{1}$ accounting for $25 \%$ of under-five mortality, $30 \%$ of childhood mortality and $11 \%$ of maternal mortality. Each year $70 \%$ of pregnant women suffer from malaria resulting in anaemia in pregnancy, abortions, stillbirths and low birth weight infants. The disease also accounts for $50-60 \%$ of outpatient consultations and $10-30 \%$ of overall hospital admissions in Nigeria. In addition to the direct health impact of malaria on the Nigerian population, the economic loss linked to the disease in this country is estimated to be about 132 billion Naira (around 878 million US \$) per year as treatment costs, loss of man-hour, to mention but a few. ${ }^{1,2}$ The disease is therefore a major public health problem in Nigeria.

Antimalarial chemotherapy has been the primary option in the fight against this menace. National drug efficacy trials conducted in 2002 in Nigeria demonstrated that the first line treatments then employed, chloroquine and sulphadoxine-pyrimethamine (SP) were no longer adequate. ${ }^{2}$ In 2005 , the highly efficacious artemisinin-based combination therapy was adopted as first-line treatment for uncomplicated malaria. ${ }^{2}$ Artemisinin-based combination therapy has since then remained the treatment of choice for uncomplicated Plasmodium falciparum malaria in Nigeria ${ }^{3}$ in line with global trend, following the recommendation of the World Health Organization (WHO) to that effect. ${ }^{4}$ Starting from February 2009 , more than 80 countries worldwide including Nigeria have adopted ACT as firstline therapy. ${ }^{3}$ Currently, five forms of ACT are recommended by the WHO, of which all are available in Nigeria. These include: i) artemether and lumefantrine (AL); ii) artesunate and amodiaquine ( $\mathrm{AS}+\mathrm{AQ})$; iii) artesunate and mefloquine (AS+MQ); iv) artesunate and sulphadoxine-pyrimethamine $(\mathrm{AS}+\mathrm{SP})$; v) dihydroartemisinin and piperaquine (DHA+PQP).

Artemisinin compounds - when used in combination with longer acting antimalarial drugs - rapidly reduce parasite densities to low levels at a time when drug levels of the longer acting drug are still maximal, thereby reducing the likelihood of parasites being exposed to suboptimal levels of the longer acting drug and limiting the emergence of resistant strains. ${ }^{5,6}$ The choice of ACT for a country or a region depends on a number of considerations. A critical element is the level of underlying resistance to the longer-acting partner drug in the combination. This is particularly important for $\mathrm{AQ}$ and SP in Africa, where both drugs have been widely used as monotherapies.

The WHO recommends that countries use ACTs, which are at least $90 \%$ effective, and introduce new forms of ACT that are at least 95\% effective after discounting reinfections
Correspondence: Paul Dienye, Department of Family Medicine, University of Port Harcourt Teaching Hospital, East-West Road, 6173 Port Harcourt, Nigeria

Tel/Fax: +234.8033.393806.

E-mail: pdienye@yahoo.com

Key words: uncomplicated malaria, Camosunate, efficacy, safety, Nigeria.

Contributions: the authors contributed equally.

Funding: the drug samples used for this study were donated by Geneith Pharmaceutical Ltd. (Oshodi, Lagos), which also funded the laboratory investigations. The company had no role in the study design, data collection and analysis, or preparation of this manuscript.

Acknowledgments: we are grateful to all the personnel of the Departments of Family Medicine and Child Health of the University of Benin Teaching Hospital who assisted the conduct of this study. We also wish to thank the haematologist, microbiologist and chemical pathologist who performed the laboratory analysis.

Received for publication: 28 March 2013

Revision received: 14 May 2013.

Accepted for publication: 17 May 2013.

This work is licensed under a Creative Commons Attribution 3.0 License (by-nc 3.0).

(C)Copyright D. Uyagu et al., 2013

Licensee PAGEPress, Italy

Healthcare in Low-resource Settings 2013; 1:e22 doi:10.4081/hls.2013.e22

and that the Day 28 efficacy of respective partner drugs alone should exceed $80 \%{ }^{7}$ Concerns have been raised over ACT including amodiaquine meeting such criteria in areas where it has been widely used as monotherapy.

The efficacy and tolerability of AS+AQ has been tested formally in several clinical trials in different epidemiological African settings. ${ }^{8-10}$

One of the AS+AQ brands in the country is Camosunate by Geneith Pharmaceutical Ltd. (Lagos, Oshodi). This study is an assessment of the efficacy and safety of Camosunate in the treatment of uncomplicated malaria conducted at the University of Benin Teaching Hospital (UBTH). Though similar studies have been conducted in other parts of Africa including Sub-Saharan Africa and Democratic Republic of Congo, ${ }^{8-10}$ none has been conducted using the Camosunate brand of ACT in South Nigeria to the best of our knowledge.

\section{Materials and Methods}

The study was conducted on patients recruited from the Departments of Family 
Medicine and Child Health of the UBTH, Benin City, Nigeria. The UBTH is a 700-bed tertiary institution made up of several clinical departments. The Department of Family Medicine has a busy general outpatient unit with a daily attendance of about 400 patients of all age groups, and is open to patients all days of the week. The Department of Child Health has consultant outpatient clinics, children's emergency room and paediatric casualty as well as in-patient facilities for children.

Benin City has a population of about 2 million people and is the capital city of Edo State, Nigeria. It is located in the tropical rain forest of South Nigeria where malaria is endemic, with high transmission rate throughout the year. Chloroquine and sulphadoxine/pyrimethamine resistance is a known problem in Benin City, like in other parts of Nigeria., ${ }^{2,11}$

This was a cross-sectional study assessing the efficacy and safety of AS+AQ. It was conducted between September 2011-2012. Malaria was diagnosed using the history of fever (axillary temperature $\geq 37.5^{\circ} \mathrm{C}$ ) and by light microscopy of thick and thin blood smears stained with $3 \%$ Giemsa for 30 min by trained microbiologists. ${ }^{12}$ Malaria parasitemia in thick films was estimated by counting asexual or sexual parasites relative to 1000 leukocytes or 500 asexual or sexual forms, whichever occurred first. A smear was declared negative when the examination of 100 thick-film fields did not reveal the presence of malaria parasites. $^{13}$

Quality control checks were performed on a random $10 \%$ sample of blood films, examined at an independent site.

Four age strata, based on the formulations of Camosunate by the manufacturers were identified prior to the study. The formulations were Camosunate adult for patients $\geq 14$ years, Camosunate junior for patients 7-13 years, Camosunate children for patients 1-6 years and Camosunate paediatric for patients less than one year of age. The proportion of patients selected from each stratum was based on the knowledge of the total patient population as obtained from the outpatient record of the UBTH. Those who gave their written informed consent were recruited into the study using simple random sampling technique (lottery method) for each stratum until the required sample size was attained. A total of 120 patients of different age groups were recruited as follows: 20 patients aged $\geq 14$ years; 30 patients aged $7-13$ years; 60 patients aged 1-6 years; and 10 patients aged $<1$ year.

The first two groups were recruited from the Department of Family Medicine, and the others from the Department of Child Health.

Inclusion criteria included: i) patients registered in UBTH; ii) patients who gave written informed consent; iii) patients with history of fever (temperature $\geq 37.5^{\circ} \mathrm{C}$ ) and with
Falciparum parasitaemia of 1000-200,000 parasites $/ \mu \mathrm{L}$; iv) patients who had not taken antimalarial drugs in the previous two weeks.

Exclusion criteria included: i) pregnant and breast feeding females because of possible toxicity to foetus and infant; ii) patients weighing less than $5 \mathrm{~kg}$, since the dynamic developmental changes experienced by infants below this weight may affect the metabolism of ingested drugs; ${ }^{14}$ iii) patients who are unable to take oral drugs; iv) patients with signs and symptoms of severe P. falciparum malaria which include convulsion, coma, jaundice and severe anaemia as published previously; ${ }^{7}$ v) very ill patients who cannot withstand the stress of the research; vi) patients with known allergy to artesunate or amodiaquine.

All recruited patients were treated with Camosunate (tablets taken with water and powdered form taken after constituting into mixture with water), in the recommended dose by the manufacturers. Each treatment was given under supervision by the researchers and the patient was observed during the first half hour. In case of vomiting, the complete dose was supplied again, and the 30 min observation was repeated. Any patient who vomited again was excluded from the study. The first dose was administered in the hospital (day 0), and patients were asked to report back on days $1,2,3,7,14,21$, and 28 , or in between as needed. The second and third doses of the drug were administered on days 1 and 2 as they reported for follow up under the observation of the researchers. Direct observation of patients by the researchers during drug intake assured that the drugs were taken, and ruled out losses due to vomiting.

The four formulations and doses of Camosunate included: i) Camosunate adult (12 tablets), each tablet containing $300 \mathrm{mg}$ amodiaquine base and $100 \mathrm{mg}$ artesunate for patients aged 14 years and over. Patients were administered 4 tablets once daily for 3 days; ii) Camosunate junior (6 tablets), each tablet containing $300 \mathrm{mg}$ amodiaquine base and $100 \mathrm{mg}$ artesunate for patients aged 7-13 years. Patients were administered 2 tablets once daily for 3 days; iii) Camosunate children (2 sachets), each containing pleasantly flavored powder containing $150 \mathrm{mg}$ amodiaquine and $50 \mathrm{mg}$ artesunate respectively for patients aged 1-6 years. Patients were administered whole contents of both sachets reconstituted in clean water once daily for 3 days; iv) Camosunate paediatric (2 sachets), each containing pleasantly flavored powder containing $75 \mathrm{mg}$ amodiaquine and $25 \mathrm{mg}$ artesunate for patients aged under 1 year. Patients were administered whole contents of both sachets reconstituted in clean water once daily for 3 days.

Patients who missed follow-up examination were traced by immediate active search or con- tacted using mobile phones. Early treatment failure was defined as the development of danger signs or severe malaria on day 1, 2 or 3 and the presence of parasitaemia on or before day 3. Late treatment failure was defined as the presence of danger signs or severe malaria in the presence of parasitaemia after day 3 or non-programmed return of the patient between days 4 to 28 due to a clinical deterioration in the presence of parasitaemia or parasitaemia on days 7, 14, 21, or 28. Success was defined as parasite clearance that was sustained through day 28. Adequate clinical-parasitological response was defined as the absence of either early or late failures. Genotyping of parasites was not done to distinguish between recrudescences and re-infections due to non-availability of equipment. The end point for efficacy and safety was day 28 . Quinine was reserved for the rescue of parasitological failures.

Safety was assessed by: i) recording treatment emergent sign/symptom (events which were not present pre-treatment or worsened with treatment); ii) measuring liver [alanine (ALT) and aspartic (AST) transaminases and bilirubin], renal functions (creatinine) and haematology [haematocrit, white blood cell (WBC) total counts]. Thirty six patients (30\%) of the study population had baseline and posttreatment measurements of these tests done (on day 0 and 28 , respectively).

Instruction was placed on the patients' records to report all cases of adverse drug reactions from the medications to the Ethics and Research Committee of the UBTH.

\section{Data analysis}

Data collected were entered into a personal computer and analyzed using Statistical Package for Social Sciences version 15. The results were presented as frequency tables, means, percentages and chi square using 95\% confidence interval and $\mathrm{P}<0.05$. Ethical approval was obtained from the ethical committee of the UBTH.

\section{Results}

The study was conducted using 120 slideproven plasmodium-infected patients with uncomplicated malaria. None of the patients was lost to follow up. Ten (8.3\%) of the patients were $<1$ year and 60 (50\%) were aged 1-6 years (Figure 1).

There were $65(54.2 \%)$ females and 55 (45.8\%) males with a ratio of 1.2:1. (Figure 2). No malaria parasite (parasite clearance) was observed in the blood film of the study populations on or before day 3 of drug administration, indicating no early treatment failure (ETF). Late treatment failure (LTF) manifested as 
recurrent parasitaemia during follow-up of five patients to day 28. The overall efficacy of Camosunate was $95.8 \%$. No patient was withdrawn due to treatment emergent signs/symptoms (TESS) or adverse drug reaction (ADR) (Table 1).

At presentation (day 0), all patients reported fever or had a measured fever in the clinic. Other malaria associated signs/symptoms on presentation were weakness (55), headache (45), vomiting (39), diarrhea (32) and nausea (12). After treatment, eleven patients (9.2\%) experienced at least one TESS which was either not present pre-treatment or worsened post-treatment. Nine patients had one TESS and two had two, making a total of thirteen TESS. Four had vomiting, two vertigo, one asthenia, two abdominal pain, one diarrhoea, one pruritus without a rash, and two headache (Table 2).

The mean values for haematocrit, $\mathrm{WBC}, \mathrm{AST}$, ALT, bilirubin and creatinine on days 0 and 28 are shown in Table 3. There was no statistical difference between the mean values $(P \geq 0.05)$

\section{Discussion}

Following the reports and documentation of reduced efficacy of chloroquine and other commonly used mono-therapies in the treatment of uncomplicated malaria, ${ }^{15}$ the World Health Organization recommended the use of arteminisin-based combination therapy (ACT) for the treatment of uncomplicated P. falciparum malaria. ${ }^{4}$ This included ArtemisininAmodiaquine combination, presenting in different brands.

In this study which aims to assess the efficacy and safety of Camosunate (a brand of $\mathrm{AS}+\mathrm{AQ}$ ) in the treatment of uncomplicated malaria, $83.3 \%$ of the subjects were below 13 years of age. This is in agreement with findings in other studies on efficacy and safety of ACTs in the management of malaria. ${ }^{8-10}$ It also
Table 1. Efficacy evaluation.

\begin{tabular}{lccccc} 
Age group & LIF & LIF & Withdrawn & MP -ve* & Total \\
$<1$ year & - & - & - & 10 & 10 \\
1-6 years & - & 2 & - & 58 & 60 \\
\hline $7-13$ year & - & 1 & - & 29 & 30 \\
$>14$ years & - & 2 & - & 18 & 20 \\
\hline Total & - & 5 & - & 115 & 120 \\
\hline
\end{tabular}

ETF, early treatment failure; LTF, late treatment failure. *No malaria parasites were detected in blood film.

Table 2. Treatment emergent signs and symptoms.

\begin{tabular}{|c|c|c|c|c|}
\hline \multirow[t]{2}{*}{ Symptoms } & \multicolumn{3}{|c|}{ Age group } & \multirow[b]{2}{*}{$>14$ years } \\
\hline & < 1 year & 1-6 years & 7-13 years & \\
\hline Abdominal pain & - & 1 & 1 & - \\
\hline Asthenia & - & - & - & 1 \\
\hline Diarrhoea & 1 & - & - & - \\
\hline Headache & - & - & 1 & 1 \\
\hline Nausea & - & - & - & - \\
\hline Pruritus & - & - & - & 1 \\
\hline Vertigo & - & - & - & 2 \\
\hline Vomiting & 1 & 2 & 1 & - \\
\hline Total & 2 & 3 & 3 & 5 \\
\hline
\end{tabular}

Table 3. Clinical laboratory values on day 0 (pre-treatment) and 28 (study end-point).

\begin{tabular}{lllcc}
\hline Laboratory test & Day & No. & Mean & SD \\
Haematocrit (\%) & 0 & 36 & 39.1 & 5.6 \\
& 28 & 36 & 38.8 & 4.8 \\
WBC $\left(x 10^{9} / \mathrm{L}\right)$ & 0 & 36 & 6.420 & 1.050 \\
& 28 & 36 & 6.887 & 0.985 \\
\hline AST (IU/L) & 0 & 36 & 40.1 & 28.7 \\
& 28 & 36 & 37.6 & 19 \\
ALT (IU/L) & 0 & 36 & 21.2 & 9.3 \\
& 28 & 36 & 18.8 & 8.7 \\
\hline Bilirubin $(\mathrm{Mmol} / \mathrm{L})$ & 0 & 36 & 6.5 & 5.4 \\
& 28 & 36 & 4.8 & 4.9 \\
Creatinine $(\mathrm{Mmol} / \mathrm{L})$ & 0 & 36 & 0.6 & 0.2 \\
& 28 & 36 & 0.7 & 0.2 \\
\hline
\end{tabular}

SD, standard deviation; WBC, white blood cell count; AST, aspartic transaminases; ALT, alanine transaminases.

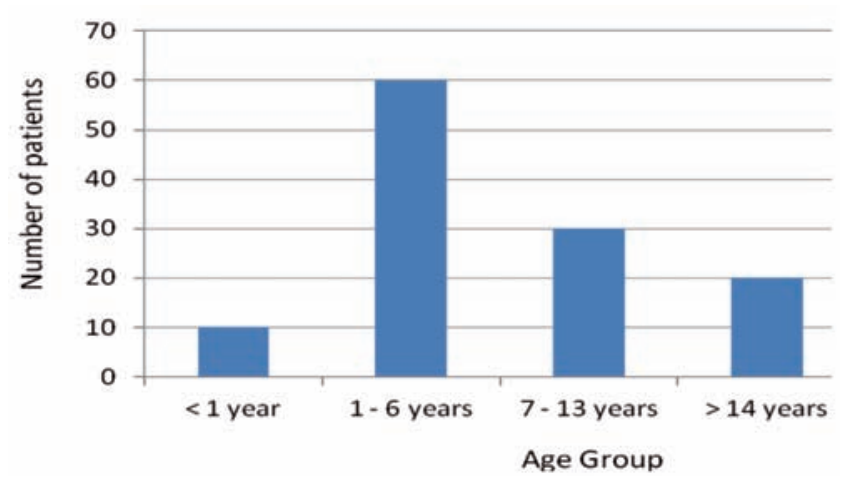

Figure 1. Age distribution of the study population.

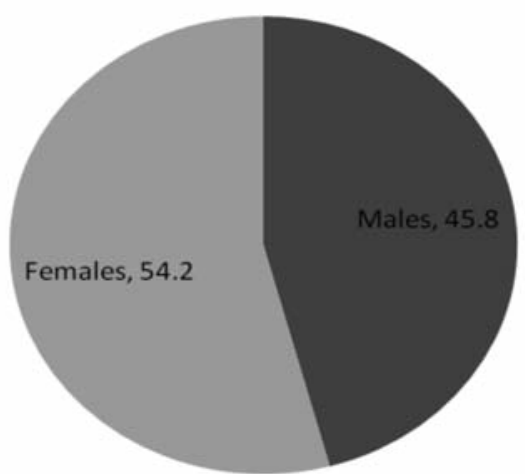

Figure 2. Gender distribution of the study population. 
underscores the fact that most of the morbidity and mortality of malaria is seen in children. ${ }^{2}$

The $100 \%$ parasite clearance observed in the blood film of the subjects by day 3 was an indication of the efficacy of the drug combination. The cure rate of $95.8 \%$ which was observed by day 28 of follow up corroborates a similar study in India in which $92.42 \%$ [real time polymerase chain reaction (PCR) corrected rate was $97.47 \%$ ] was observed. ${ }^{18}$ The positive malaria parasite smears observed in five patients (4.2\%) by day 28 could not be labeled as recrudescence or re-infection since genotyping of the parasites was not done due to non-availability of appropriate equipments. Similar observation of late treatment failure was reported in Ghana ${ }^{12}$ but higher than the $2.5 \%$ finding in India. ${ }^{16}$ Treatment was well tolerated as testified by the fact that no case was withdrawn because of severe TESS or adverse drug reaction. There were, however, thirteen TESS experienced by eleven (9.2\%) of the study group. This is higher than the finding in India in which $2.5 \%$ was reported. ${ }^{16}$ Vomiting was the most common TESS observed in this study. This may indicate gastric irritation caused by the study medication. The tolerability of this drug which was proven by no withdrawal from this study may have been due to the enlightenment of the subjects and their mothers or care givers on the known usual side effects of amodiaquine.

Amodiaquine-associated toxicities (hepatitis or severe leucopenia) have been reported in the past when it was used as prophylaxis in travelers. ${ }^{17}$ These and other toxic reactions were not observed among the patients in this study as manifested by the absence of statistically significant difference between the mean values of liver AST, ALT and bilirubin, renal creatinine and haematology (haematocrit, WBC total count) on days 0 and 28. However, the number of closely monitored patients was too small to detect rare toxicities. The good compliance, resulting in good treatment outcome in this study could be attributed to the simple dose regimen as recommended by the manufacturers. It is generally accepted that making treatments easier to understand and use by patients or their care givers results in better compliance and that using fixed-dose combinations enhances this. ${ }^{17,18}$

\section{Limitations}

Microscopy is the gold standard and mainstay for measuring parasitemia. However, it requires considerable expertise, has low sensitivity and is labour intensive. Even with these disadvantages, microscopy was used to detect parasite clearance due to the non-availability of more modern equipments such as real time PCR and delta delta cycle threshold (DDCT) calculation. ${ }^{19}$ This did not allow comparison of our results with others in which modern methods were used.

Another limitation imposed on the study was the paucity of drugs and reagents. This influenced the utilization of a sample size not based on power analysis in this pilot study. A larger sample size may have given better results.

\section{Conclusions}

Antimalarial medicines have an important role to play in reducing malaria transmission and curtailing the spread of drug resistant parasites. Camosunate provided an efficacious treatment of falciparum malaria in Nigerian patients. It was well tolerated and it exhibited no adverse drug reactions.

\section{References}

1. Federal Republic of Nigeria. National antimalarial treatment policy. Abuja, Nigeria: Federal Republic of Nigeria, Ministry of Health National Malaria and Vector Division ed.; 2005. Available from: http://apps.who.int/medicinedocs/documents/s18401en/s18401en.pdf

2. Federal Republic of Nigeria. Strategic plan 2009-2013. A road map to malaria control in Nigeria. Abuja, Nigeria: Federal Republic of Nigeria, Ministry of Health, National Malaria Control Program; 2009.

3. Federal Republic of Nigeria. National antimalarial treatment policy. Abuja, Nigeria: Federal Republic of Nigeria, Ministry of Health ed.; 2005.

4. WHO. Antimalarial drug combination therapy: report of a WHO technical consultation. Geneva: World Health Organisation ed.; 2001.

5. WHO. Global antimalarial drug policy database, Africa. Available from: http://www.who.int/malaria/am_drug_policies_by_region_afro/en/

6. White NJ. Delaying antimalarial drug resistance with combination chemotherapy. Parassitologia 1999;44:301-8.

7. WHO. Guidelines for the treatment of malaria. Geneva: World Health Organisation ed.; 2010. Available from: http://whqlibdoc.who.int/publications/2010/9789241547925_eng.pdf

8. Espié E, Lima A, Atua B, et al. Efficacy of fixed-dose combination artesunate-amodiaquine versus artemether-lumefantrine for uncomplicated childhood Plasmodium falciparum malaria in Democratic Republic of Congo: a randomized non-inferiority trial. Malaria J 2012;11:174.

9. Zwang J, Olliaro P, Barennes $\mathrm{H}$, et al. Efficacy of artesunate-amodiaquine for treating uncomplicated malaria in subSaharan Africa: a multi-centre analysis. Malaria J 2009;8:203.

10. Brasseur P, Agnamey P, Gaye O, et al. Efficacy and safety of artesunate plus amodiaquine in routine use for the treatment of uncomplicated malaria in Casamance, Southern Senegal. Malaria J 2007;6:150.

11. Ogungbamigbe T0, Ojurongbe 0 , Ogunro PS, et al. Chloroquine resistant Plasmodium falciparum malaria in 0sogbo Nigeria: efficacy of amodiaquine + sulfadoxine-pyrimethamine and chloroquine + chlorpheniramine for treatment. Mem I 0swaldo Cruz 2008;103:78-84.

12. Koram KA, Quaye L, Abuaku B. Efficacy of Amodiaquine/Artesunate combination therapy for uncomplicated malaria in children under five years in Ghana. Ghana Med J 2008;42:55-60.

13. Gbotosho G0, Sowunmi A, Okuboyejo TM, et al. Therapeutic efficacy and effects of Artemether-Lumefantrine and ArtesunateAmodiaquine coformulated or copackaged on malaria-associated anemia in children with uncomplicated plasmodium falciparum malaria in Southwest Nigeria. Am J Trop Med Hyg 2011;84:813-9.

14. Kearns GL, Abdel-Rahman SM, Alander SW, et al. Developmental pharmacology: drug disposition, action, and therapy in infants and children. New Engl J Med 2003;349:1157-67.

15. Sowunmi A, Ayede AI, Falade AG, et al. Randomized comparison of chloroquine and amodiaquine in the treatment of acute, uncomplicated, Plasmodium falciparum malaria in children. Ann Trop Med Parasit 2001;95:549-58.

16. Anvikar AR, Sharma B, Shahi BH, et al. Artesunate-amodiaquine fixed dose combination for the treatment of Plasmodium falciparum malaria in India. Malaria $\mathrm{J}$ 2012;11:97.

17. Taylor WRJ, White NJ. Antimalarial drug toxicity. A review. Drug safety 2004;27:2561 .

18. Ratsimbasoa A, Randrianarivelojosia M, Millet $\mathrm{P}$, et al. Use of a pre-packadged chloroquine for the home management of presumed malaria in Malagasy children. Malaria J 2006;14:79.

19. Beshir K, Sutherland C. Measuring the efficacy of anti-malarial drugs in vivo: quantitative PCR measurement of parasite clearance. Malaria J 2010;9:312. 